\title{
Normative Database of Peripapillary Vessel Density Measured by Optical Coherence Tomography Angiography and Correlation Study
}

José Ignacio Fernández-Vigo, Bachar Kudsieh, Hang Shi, Lucía De-PabloGómez-de-Liaño, Irene Serrano-Garcia, José María Ruiz-Moreno, José María Martínez-de-la-Casa, Julián García-Feijóo \& José ngel Fernández-Vigo

To cite this article: José Ignacio Fernández-Vigo, Bachar Kudsieh, Hang Shi, Lucía De-PabloGómez-de-Liaño, Irene Serrano-Garcia, José María Ruiz-Moreno, José María Martínez-de-la-Casa, Julián García-Feijóo \& José ngel Fernández-Vigo (2020): Normative Database of Peripapillary Vessel Density Measured by Optical Coherence Tomography Angiography and Correlation Study, Current Eye Research, DOI: 10.1080/02713683.2020.1744164

To link to this article: https://doi.org/10.1080/02713683.2020.1744164

Accepted author version posted online: 18 Mar 2020.

Published online: 27 Mar 2020.

Submit your article to this journal

Цll Article views: 29

Q View related articles $\sqsubset$

View Crossmark data $־$ 


\title{
Normative Database of Peripapillary Vessel Density Measured by Optical Coherence Tomography Angiography and Correlation Study
}

\author{
José Ignacio Fernández-Vigo $\mathbb{1}^{\mathrm{a}, \mathrm{b}}$, Bachar Kudsieh ${ }^{\mathrm{b}, \mathrm{c}}$, Hang Shi ${ }^{\mathrm{a}, \mathrm{b}}$, Lucía De-Pablo-Gómez-de-Liaño ${ }^{\mathrm{b}, \mathrm{d}}$, Irene Serrano- \\ Garciae, José María Ruiz-Moreno o,f, José María Martínez-de-la-Casaa , Julián García-Feijóoa, and José ngel Fernández-Vigo b,g \\ aDepartment of Ophthalmology, Hospital Clínico San Carlos, Instituto de Investigación Sanitaria (IdISSC), OFTARED, Madrid, Spain; bOphthalmology, \\ Centro Internacional de Oftalmología Avanzada, Madrid, Spain; 'Department of Ophthalmology, Hospital Puerta de Hierro Majadahonda, Madrid, \\ Spain; 'Department of Ophthalmology, Hospital 12 de Octubre, Madrid, Spain; eDepartment of Preventive Medicine, Instituto de Investigación \\ Sanitaria San Carlos (IdISSC), Hospital Universitario Clínico San Carlos, Madrid, Spain; fDepartment of Ophthalmology, Castilla La Mancha University, \\ Albacete, Spain; ${ }^{9}$ Department of Ophthalmology, Universidad de Extremadura, Badajoz, Spain
}

\begin{abstract}
Purpose: To provide a normative data set of swept-source optical coherence tomography angiography (SS-OCTA) peripapillary vessel density (VD) measurements and assess correlations with age, gender, disc area and axial length $(\mathrm{AL})$.

Material and Methods: This was an observational cross-sectional study conducted on 346 right eyes of healthy subjects. Peripapillary SS-OCTA VD measurements were obtained in the retinal superficial capillary plexus (SCP), deep capillary plexus (DCP), and choriocapillaris (CC) in all four quadrants using the Triton device (Topcon, Nagoya, Japan). AL measurements were made by optical biometry.

Results: Mean participant age was $37.7 \pm 19.8$ years (range $5-83), A L$ was $23.9 \pm 1.3 \mathrm{~mm}(21-27)$, and $59 \%$ were women. Mean VDs for all quadrants were $57.2 \pm 5.7 \%$ for SCP $(52.4 \pm 4.0 \%-62.1 \pm 4.2 \%)$, $46.3 \pm 6.5 \%$ for DCP $(41.9 \pm 5.8 \%-49.7 \pm 4.8 \%)$ and $60.8 \pm 7.4 \%$ for CC $(53.4 \pm 5.8 \%-65.7 \pm 5.5 \%)$. Negative correlation was detected between SCP VD and age $(R=-0.264 ; P<.001)$, while correlation between DCP densities and age was positive $(R=0.202 ; P=.002)$. No associations were detected between peripapillary VD and gender $(P \geq 0.087)$ and negative correlation was found with $A L$ and disc area only in the CC (P $\leq 0.004)$. In a subgroup analysis of 33 individuals, we noted no differences in VD between fellow eyes ( $P \geq 0.139$ ).

Conclusion: Peripapillary VD showed wide individual variation when measured with the Triton SS-OCTA in the different retinal plexuses and CC. While correlations were observed with age, no relationships emerged with gender and only CC showed negative correlation with AL and disc area.
\end{abstract}

\section{ARTICLE HISTORY}

Received 11 November 2019

Revised 16 February 2020

Accepted 10 March 2020

\section{KEYWORDS}

Peripapillary vessel density; optic nerve head; optical coherence tomography angiography; glaucoma retinal vascular plexus

\section{Introduction}

Vascular dysfunction plays a key role in the pathogenesis of glaucoma, as many studies have shown. ${ }^{1-3}$ However, knowledge about the relationship between abnormal ocular perfusion and glaucoma is still scarce mainly because of limitations in determining optic nerve head $(\mathrm{ONH})$ blood flow. ${ }^{4,5}$

By optical coherence tomography angiography (OCTA), a recently introduced technique, the microcapillaries of the $\mathrm{ONH}$ and peripapillary region can be easily visualized without the need for dye injection. ${ }^{4-10}$ This technique based on comparing B-scans via OCTA algorithms is able to detect red blood cell motions and can therefore be used to visualize microvascular flow.

Among the quantitative metrics used to describe the state of the $\mathrm{ONH}$ and peripapillary vessels offered by current OCTA devices, one of the most popular is vessel density (VD) ${ }^{8,9,11-13}$ So far, OCTA has proved useful to evaluate ONH VD in patients with suspected or confirmed glaucoma. ${ }^{5}$ The clinical relevance of taking peripapillary VD measurements is that the amount of VD reduction produced seems related to the extent of functional loss in glaucoma.
Using this technique, Jia et al. were the first to describe a reduction in $\mathrm{ONH}$ and peripapillary $\mathrm{VD}$ in pre-perimetric and primary open-angle glaucoma (POAG) compared to controls. ${ }^{4}$ Interestingly, in patients with suspected or confirmed glaucoma, Triolo et al. observed evident structural damage in both peripapillary and macular vessel networks. ${ }^{14}$ Moreover, OCTA has been successfully used to distinguish between healthy subjects, patients with ocular hypertension, glaucoma suspects, and patients with POAG. ${ }^{14,15}$ Diminished VD has also been reported in other types of glaucoma such as normal-tension, ${ }^{6,7}$ primary angle-closure ${ }^{16,17}$ or exfoliation. ${ }^{18}$ Several studies have also detected strong correlation between OCTA parameters and the thickness of the retinal nerve fiber layer (RNFL) $)^{5,19}$ or ganglion cell complex..$^{20}$ Further, peripapillary VD has been correlated moderately to highly with visual field (VF) parameters. ${ }^{4,10,21}$

As this technology is relatively new, there are still few large normative OCTA databases and little is also known about the influence of or correlation between different parameters and peripapillary or $\mathrm{ONH} \mathrm{VD}^{8,11}$ Thus, there is a need for 
normative VD data before OCTA technology can be routinely used in clinical practice and trials to guide the diagnosis and management of glaucoma. We will then be able to compare these reference data with measurements made in the different types of glaucoma for an effective diagnosis and to detect glaucoma progression ${ }^{4-7,22,23}$ ideally in early disease stages but also in advanced stage disease. ${ }^{13,15}$

With this purpose in mind, the present study was designed to provide normative peripapillary data of the retinal plexus and choriocapillaris (CC) obtained through SS-OCTA in a large population of healthy individuals. Once these data were compiled, we examined correlations between VD measurements and the factors gender, age, disc area and axial length $(\mathrm{AL})$.

\section{Methods}

The participants of this cross-sectional study were 346 healthy volunteers. Patients attending the Centro Internacional de Oftalmologia Avanzada (Madrid, Spain) for a routine eye examination were enrolled over the period February 1, 2018 to January 10, 2019. Once it was ensured that all the established inclusion criteria were met, candidates were invited to participate. After explaining the nature of the study protocol and its objectives, participants gave their written consent to participate. The study protocol was in line with the Declaration of Helsinki and received Institutional Review Board approval.

For enrollment it was required that subjects be healthy Caucasian (white race) with an intraocular pressure equal to or under $21 \mathrm{~mm} \mathrm{Hg}$ in both eyes, and an AL of 21 to $27 \mathrm{~mm}$ (mean AL among our patients is $24 \mathrm{~mm}$ ). Subjects were excluded if they had a self-proclaimed systemic or eye disease (or one found in the ophthalmological examination), ocular media opacification, had undergone previous eye surgery or were taking any systemic medication. Subjects were also excluded if their corresponding images had a motion artifact consisting of a discontinuous vessel pattern or discontinuous disc boundary. The presence of strabismus and ocular torsion were ruled out in the examination and in the fundus photograph. Measurements for the right eye only were analyzed.

Participants were subjected to a full ophthalmologic examination and OCTA on the same day. The parameters recorded were visual acuity and refraction, and slit-lamp biomicroscopy and posterior segment ophthalmoscopy findings. Also recorded were age, gender and AL (IOL master 500; Carl Zeiss Jena, Germany). To assess inter-eye differences, data for the left eye were also considered in the first 33 participants examined.

For the VD measurements, we used the Triton OCTA device (Topcon, Tokyo, Japan). The instrument's working conditions were: central wavelength $1,050 \mathrm{~nm}$, axial resolution $8 \mu \mathrm{m}$, transverse resolution $20 \mu \mathrm{m}$, and scan speed 100,000 A-scans/s. All OCTA measurements were made after pupil dilation (Colircusi tropicamide $10 \mathrm{mg} / \mathrm{ml}$, Alcon Laboratories, Inc., Fort Worth, TX) by a well-trained examiner (HS) with the subject sitting and looking at a fixed point. Only images of sufficient quality (signal strength intensity (SSI) $>40$ ) were accepted.
The algorithm used by the Triton device was OCTA-ratio analysis (OCTARA, full-spectrum ratio-based amplitude ratio analysis) keeping the full-spectrum intact and preserving axial resolution. Vessel density was defined as the percentage of vessel area showing blood flow out of the total area measured and determined through adaptive thresholding binarization. Selecting the mode "angio macula", a $6 \times 6 \mathrm{~mm}$ scan was centered manually at the $\mathrm{ONH}$ and VD measurements made in the peripapillary quadrants: superior, inferior, nasal and temporal. These quadrants were identified using an ETDRS grid overlay comprising the two inner rings. Vessel densities were obtained after applying the device's projections removal algorithm to the deep capillary plexus (DCP) and CC images. Images were first checked for good segmentation and the disc area was measured.

Peripapillary VD measurements were automatically segmented by the software algorithm (version 2014.2.0.65) for the superficial capillary plexus (SCP), DCP and CC. According to these default settings, the SCP network runs from $2.6 \mu \mathrm{m}$ below the internal limiting membrane to $15.6 \mu \mathrm{m}$ below the inner plexiform layer (IPL); DCP runs from $15.6 \mu \mathrm{m}$ to $70.2 \mu \mathrm{m}$ below the IPL; and CC runs from the Bruch membrane to $10.4 \mu \mathrm{m}$ beneath it (Figure 1). This determined that the nerve fiber layer plexus (NFLP; traditionally known as the radial peripapillary capillaries) and ganglion cell layer plexus (GCLP) were included in the SCP by the software employed.

\section{Statistical analysis}

For the statistical treatment of data, we used the software package SPSS ${ }^{\circ}$ (Statistical Package for Social Sciences, v18.0; SPSS Inc., Chicago, IL, USA). Data are provided as the mean and standard deviation for quantitative variables, and frequency distributions for qualitative variables. To establish reference VD values for the different plexuses, percentile distributions (P5 and P95) were estimated. The KolmogorovSmirnov test was used to assess the normality of data distribution. We used the Pearson's correlation procedure to assess correlations between peripapillary VD and the remaining variables and between data for right and left eyes in the subgroup examined. SCP versus DCP VD and right versus left eye measurements were compared using a paired t-test. We also conducted a multivariate stepwise linear regression analysis to identify the main factors determining a greater or lesser peripapillary VD. Significance was set at $p<.05$.

\section{Results}

Mean participant age was $37.7 \pm 19.8$ years (range 5- 83); $59 \%$ were women. Mean AL was $23.9 \pm 1.3 \mathrm{~mm}(21-27)$, mean optic disc area was $2.09 \pm 0.38 \mathrm{~mm}^{2}(1.41-3.27)$ and scan quality was $63.0 \pm 6.8$ SSI (41-77). All variables studied were normally distributed.

Mean peripapillary VD was $57.2 \pm 5.7 \%$ for SCP, $46.3 \pm 6.5 \%$ for DCP and $60.8 \pm 7.4 \%$ for CC. Mean VDs for SCP were significantly higher than for DCP $(P<.001$ for all quadrants) (Table 1). 


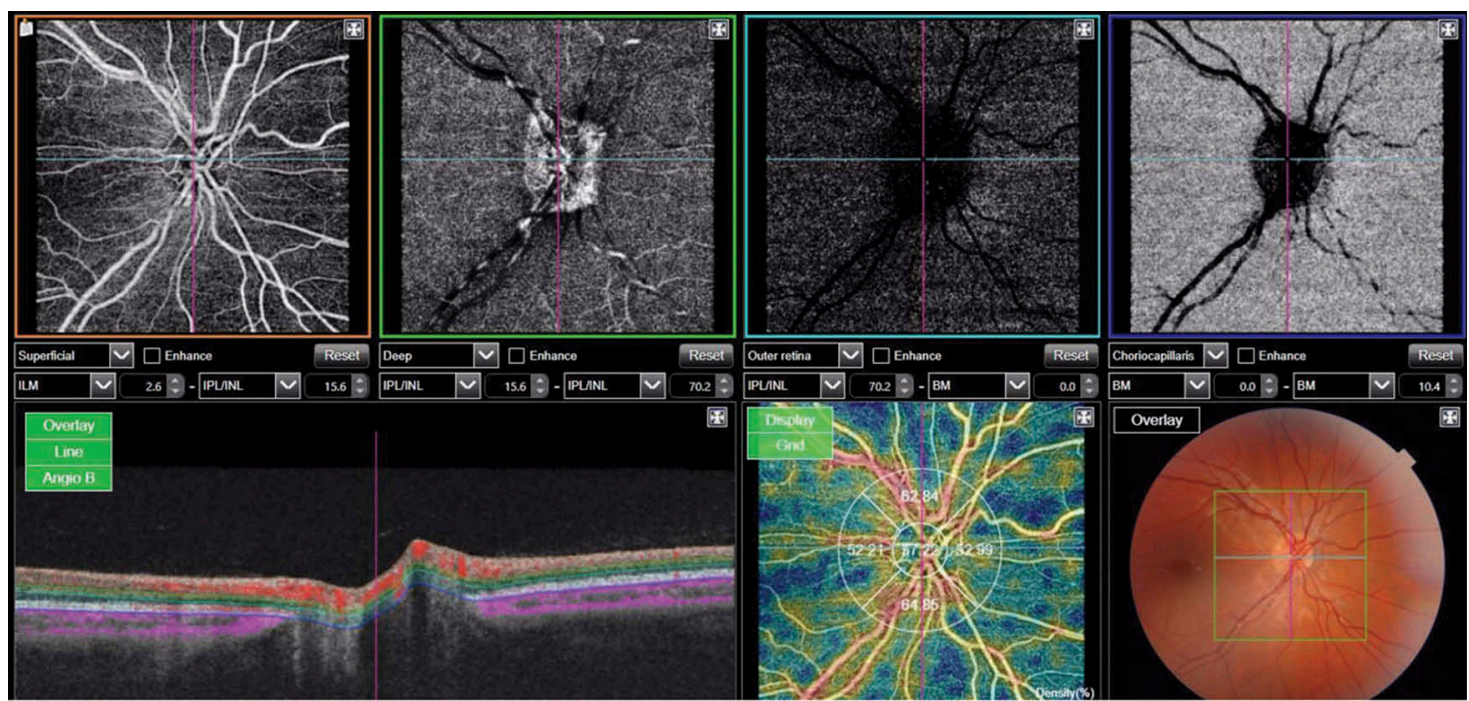

Figure 1. Example of a $6 \times 6 \mathrm{~mm}$ optic nerve head $(\mathrm{ONH})$ vessel density map showing measurements taken across the peripapillary quadrants segmented into the superficial capillary plexus (SCP), deep capillary plexus (DCP) and choriocapillaris (CC) layer.

Table 1. Swept source optical coherence tomography angiography (SS-OCTA) peripapillary vessel densities measured in the different retinal plexuses and choriocapillaris expressed as percentages (mean \pm standard deviation and range) and percentile distributions (P5 and P95).

\begin{tabular}{lccc}
\hline Peripapillary VD & $\mathrm{SCP}^{\mathrm{a}}$ & $\mathrm{DCP}^{\mathrm{b}}$ & $\mathrm{CC}^{\mathrm{C}}$ \\
\hline Whole & $57.2 \pm 5.7$ & $46.3 \pm 6.5$ & $60.8 \pm 7.4$ \\
& $(37.6-74.6)$ & $(26.7-70.5)$ & $(29.9-79.9)$ \\
& $\mathrm{P} 5-\mathrm{P} 95:$ & $\mathrm{P} 5-\mathrm{P} 95:$ & $\mathrm{P} 5-\mathrm{P} 95:$ \\
Superior & $48.1-65.9$ & $35.6-56.8$ & $48.4-72.2$ \\
& $59.9 \pm 4.3$ & $41.9 \pm 5.8$ & $58.8 \pm 5.3$ \\
& $(42.7-74.6)$ & $(26.7-65.3)$ & $(35.9-71.1)$ \\
& $\mathrm{P}-\mathrm{P} 95:$ & $\mathrm{P} 5-\mathrm{P} 95:$ & $\mathrm{P} 5-\mathrm{P} 95:$ \\
Inferior & $52.7-66.0$ & $33.3-51.2$ & $49.1-66.9$ \\
& $62.1 \pm 4.2$ & $45.3 \pm 7.1$ & $53.4 \pm 5.8$ \\
& $(38.6-71.5)$ & $(28.6-66.7)$ & $(30.2-72.6)$ \\
& $\mathrm{P} 5-\mathrm{P} 95:$ & $\mathrm{P} 5-\mathrm{P} 95:$ & $\mathrm{P} 5-\mathrm{P} 95:$ \\
Temporal & $54.9-68.2$ & $34.3-58.2$ & $44.0-62.0$ \\
& $52.4 \pm 4.0$ & $48.2 \pm 5.2$ & $65.4 \pm 4.8$ \\
& $(37.6-63.6)$ & $(32.1-70.5)$ & $(52.7-79.9)$ \\
Nasal & $\mathrm{P} 5-\mathrm{P} 95:$ & $\mathrm{P} 5-\mathrm{P} 95:$ & $\mathrm{P} 5-\mathrm{P} 95:$ \\
& $45.3-58.7$ & $40.6-56.8$ & $57.8-73.2$ \\
& $54.2 \pm 3.7$ & $49.7 \pm 4.8$ & $65.7 \pm 5.5$ \\
& $(42.6-69.0)$ & $(36.5-68.5)$ & $(29.9-76.0)$ \\
& $\mathrm{P} 5-\mathrm{P} 95:$ & $\mathrm{P} 5-\mathrm{P} 95:$ & $\mathrm{P} 5-\mathrm{P} 95:$ \\
& $48.1-59.9$ & $41.5-57.7$ & $55.8-73.8$ \\
\hline
\end{tabular}

${ }^{\mathrm{a}} \mathrm{SCP}=$ superficial capillary plexus; ${ }^{\mathrm{b}} \mathrm{DCP}=$ deep capillary plexus; ${ }^{\mathrm{C}} \mathrm{CC}=$ choriocapillaris.

In the SCP, vertical peripapillary VDs were higher than horizontal measurements $(P<.001)$. For CC, higher mean peripapillary VDs were observed in the horizontal (nasal $65.7 \pm 5.5 \%$ and temporal $65.4 \pm 4.8 \%$ ) than vertical quadrants (superior $58.8 \pm 5.6 \%$ and inferior $53.6 \pm 5.9 \%$; $P<.001$ ).

When comparing vessel densities across the different peripapillary plexuses (Table 2), correlation was observed between SCP and DCP (from $\mathrm{R}=0.169$ to 0.516; $\mathrm{p} \leq 0.002)$. However, DCP and CC showed weak negative correlation between the inferior and nasal quadrants $(\mathrm{R}=-0.256$ and $\mathrm{R}=-0.162 ; P<.001)$. In the subgroup of 33 subjects, no differences were observed in VD between fellow eyes $(P \geq 0.139)$.

We detected negative correlation between peripapillary SCP VD and age (from $R=-0.264$ to $R=-0.166$ for all
Table 2. Correlations between peripapillary vessel density (VD) measured in the different vascular plexuses (superficial capillary plexus $=$ SCP; deep capillary plexus = DCP; and choriocapillaris $=$ CC).

\begin{tabular}{llc}
\hline Peripapillary VD & Parameter & Correlation $(p$ value $)$ \\
\hline Superior & SCP - DCP & $0.239(P<.001)$ \\
& SCP - CC & $0.076(P=.162)$ \\
Inferior & DCP - CC & $-0.052(P=.340)$ \\
& SCP - DCP & $0.169(P=.002)$ \\
Temporal & SCP - CC & $0.021(P=.695)$ \\
& DCP - CC & $-0.256(P<.001)$ \\
Nasal & SCP - DCP & $0.385(P<.001)$ \\
& SCP - CC & $0.387(P<.001)$ \\
& DCP - CC & $0.055(P=.309)$ \\
& SCP - DCP & $0.516(P<.001)$ \\
& SCP - CC & $-0.050(P=.360)$ \\
& DCP - CC & $-0.162(P=.003)$ \\
\hline
\end{tabular}

quadrants; $\mathrm{P} \leq 0.002)$, and positive correlation between $\mathrm{DCP}$ vessel densities and age in the vertical quadrants $(R=0.202$ and 0.240 for the superior and inferior quadrants respectively; $P \leq 0.002$ ). (Table 3 and Figure 2). The association between age and VD was also analyzed in the subgroup of patients $\leq 18$ years of age $(\mathrm{n}=60)$ but no significant correlation was found (SCP R = $-0.123, P=.329$; DCP R $=0.053, P=.673$; CC R $=-0.025, P=.841$; Spearman test).

For disc area, we detected no significant correlation with SCP VD, but weak positive association with DCP VD was found $(\mathrm{R} \leq 0.236)$. On the contrary, negative correlation was noted between CC VD and disc area ( $\mathrm{R}=-0.349$ to -0.273 ; $\mathrm{P} \leq 0.001)$.

In our multivariate regression analysis, age remained as a determinant of peripapillary VD $(P<.001)$ for all plexuses. However, no association was found between peripapillary VD and gender $(P \geq 0.087)$ regardless of the network examined. Only CC VD showed correlation with AL $(P=.004)$. Disc area persisted as a significant determinant of DCP- and CC VD (Table 4).

\section{Discussion}

To confidently employ OCTA technology for quantitative metrics in clinical research and practice, normative peripapillary VD data are needed. Once we acquire these reference data, we will be able to 
Table 3. Correlation between peripapillary vessel density (VD) and age or disc area $(\mathrm{N}=346)$. Correlation between peripapillary VD in the right and fellow eye is also shown (data recorded in 33 subjects).

\begin{tabular}{|c|c|c|c|c|c|}
\hline $\begin{array}{l}\text { Peripapillary } \\
\text { VD }\end{array}$ & Quadrant & $\begin{array}{c}\text { Age } \\
\text { (years) }\end{array}$ & $\begin{array}{c}\text { Disc area } \\
\left(\mathrm{mm}^{2}\right)\end{array}$ & $\begin{array}{c}\text { Fellow eye } \\
(\mathrm{N}=33)\end{array}$ & $\begin{array}{c}\text { Fellow } \\
\text { eye } \\
\text { paired } \\
\text { t test } \\
(\mathrm{N}=33)\end{array}$ \\
\hline \multirow[t]{4}{*}{$\mathrm{SCP}^{\mathrm{a}}$} & Superior & $\begin{array}{c}\mathrm{R}=-0.264 \\
P<.001\end{array}$ & $\begin{array}{c}\mathrm{R}=-0.005 \\
P=.931\end{array}$ & $R=-0.100$ & $P=.572$ \\
\hline & Inferior & $\begin{array}{c}\mathrm{R}=-0.247 \\
P<.001\end{array}$ & $\begin{array}{c}\mathrm{R}=-0.064 \\
P=.248\end{array}$ & $R=0.084$ & $P=.637$ \\
\hline & Temporal & $\begin{array}{c}\mathrm{R}=-0.189 \\
P<.001\end{array}$ & $\begin{array}{c}\mathrm{R}=0.147 \\
P=.088\end{array}$ & $R=-0.242$ & $P=.167$ \\
\hline & Nasal & $\begin{array}{c}\mathrm{R}=-0.166 \\
P=.002\end{array}$ & $\begin{array}{c}\mathrm{R}=0.073 \\
P=.188\end{array}$ & $R=-0.218$ & $P=.215$ \\
\hline \multirow[t]{4}{*}{$D C P^{b}$} & Superior & $\begin{array}{c}\mathrm{R}=0.202 \\
P=.002\end{array}$ & $\begin{array}{c}\mathrm{R}=0.236 \\
P<.001\end{array}$ & $R=0.075$ & $P=.673$ \\
\hline & Inferior & $\begin{array}{c}\mathrm{R}=0.240 \\
P=.000\end{array}$ & $\begin{array}{l}\mathrm{R}=0.186 \\
P<.001\end{array}$ & $R=0.052$ & $P=.771$ \\
\hline & Temporal & $\begin{array}{c}R=-0.057 \\
P=.292\end{array}$ & $\begin{array}{c}\mathrm{R}=-0.003 \\
P=.157\end{array}$ & $R=-0.356$ & $P=.139$ \\
\hline & Nasal & $\begin{array}{c}\mathrm{R}=-0.068 \\
P=.213\end{array}$ & $\begin{array}{c}\mathrm{R}=0.178 \\
P<.001\end{array}$ & $R=-0.019$ & $P=.916$ \\
\hline \multirow[t]{4}{*}{$\mathrm{CC}^{\mathrm{C}}$} & Superior & $\begin{array}{c}\mathrm{R}=0.040 \\
P=.464\end{array}$ & $\begin{array}{c}\mathrm{R}=-0.325 \\
P<.001\end{array}$ & $R=-0.097$ & $P=.584$ \\
\hline & Inferior & $\begin{array}{c}\mathrm{R}=-0.126 \\
P=.060\end{array}$ & $\begin{array}{c}\mathrm{R}=-0.341 \\
P<.001\end{array}$ & $R=0.005$ & $P=.998$ \\
\hline & Temporal & $\begin{array}{c}\mathrm{R}=-0.352 \\
P=.052\end{array}$ & $\begin{array}{c}\mathrm{R}=-0.349 \\
P<.001\end{array}$ & $R=-0.056$ & $P=.752$ \\
\hline & Nasal & $\begin{array}{c}\mathrm{R}=-0.066 \\
P=.226\end{array}$ & $\begin{array}{c}\mathrm{R}=-0.273 \\
P<.001\end{array}$ & $R=0.137$ & $P=.438$ \\
\hline
\end{tabular}

compare them with VD measurements obtained in the different types of glaucoma and optic nerve diseases to detect different abnormalities, ideally in early disease stages. ${ }^{4-7,13,15,22,23}$

In the present study, we used an SS-OCTA device equipped with automatic software to quantify peripapillary VD not only in the retinal SCP, but also in the DCP and in the CC, obtaining a mean VD of $57.2 \pm 5.7 \%, 46.3 \pm 6.5 \%$ and $60.8 \pm 7.4 \%$ respectively. In the SCP, VD was greater in the vertical (superior $59.9 \pm 4.3 \%$, inferior $62.1 \pm 4.2 \%$ ) than horizontal quadrants (temporal $52.4 \pm 4.0 \%$, nasal $54.2 \pm 3.7 \%$ ), probably because of the presence of the larger retinal vessels.

In an interesting study, Bazvand et al. generated a normal quantitative database of peripapillary VD measurements made

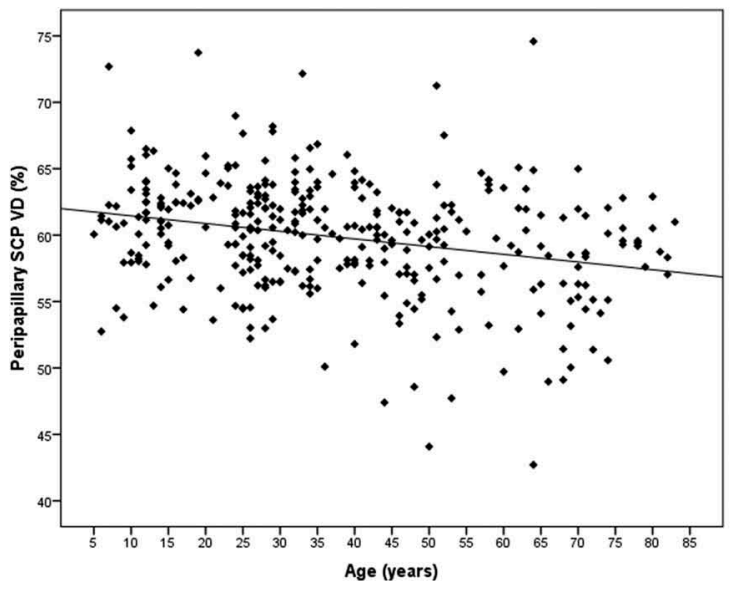

Table 4. Multivariate regression analysis of factors determining a greater or lesser peripapillary vessel density (VD).

\begin{tabular}{lcc}
\hline Dependent variable: peripapillary SCP ${ }^{\mathrm{a}}$ VD & Unstandarized beta & $\mathrm{p}$ \\
\hline Constant & 60.447 & .000 \\
Age (years) & -0.064 & $<.001^{*}$ \\
Gender (male) & -0.035 & .954 \\
Axial length (mm) & -0.134 & .583 \\
Scan quality (signal strength intensity) & 0.086 & $.041^{*}$ \\
Disc area (mm ${ }^{2}$ ) & 0.000 & 1.000 \\
\hline Dependent variable: peripapillary DCP ${ }^{\mathrm{b}}$ VD & Unstandarized beta & $\mathrm{p}$ \\
\hline Constant & 33.611 & .001 \\
Age (years) & 0.078 & $<.001^{*}$ \\
Gender (male) & -0.986 & .234 \\
Axial length (mm) & -0.102 & .760 \\
Scan quality (signal strength intensity) & 0.044 & .442 \\
Disc area (mm ${ }^{2}$ ) & 2.874 & $.006^{*}$ \\
\hline Dependent variable: peripapillary CC ${ }^{\mathrm{c}}$ VD & Unstandarized beta & $\mathrm{p}$ \\
\hline Constant & 43.833 & .000 \\
Age (years) & -0.061 & $<.001^{*}$ \\
Gender (male) & -0.347 & .648 \\
Axial length (mm) & -0.862 & $.004^{*}$ \\
Scan quality (signal strength intensity) & -0.029 & .584 \\
Disc area (mm ${ }^{2}$ ) & -5.576 & $<.001^{*}$ \\
\hline${ }^{a}$ SCP = superficial capillary $\quad$ plexus; ${ }^{\mathrm{b}}$ DCP & deep capillary & plexus; \\
${ }^{C}$ CC = choriocapillaris. & &
\end{tabular}

using the RTVue XR Avanti (Optovue, Fremont, CA, USA) in 79 eyes; the mean VD reported was $60.5 \pm 2.4 \% .{ }^{11}$ In another study in 181 eyes of 107 subjects, using the same device (RTVue XR Avanti), Rao et al. detected a median peripapillary VD of $62.7 \% .^{8}$ Also in accordance with our findings for the SCP $(57.2 \pm 4.0 \%)$, Akagi et al. observed a peripapillary VD in 21 normal eyes of $55.2 \pm 3.4 \%,{ }^{21}$ while Falavarjani et al. obtained a mean of $60.1 \pm 2.8 \%$ in 58 healthy individuals. ${ }^{24}$ In the study of Bazvand et al., the highest VD was recorded in the inferotemporal subfield $(62.5 \pm 3.8 \%) .{ }^{11}$ Similarly, Rao et al. also recorded higher densities for the inferotemporal and superotemporal subfields (both $66.8 \%$ ). ${ }^{8}$ In our study, the highest VD for the SCP was recorded in the inferior quadrant $(62.1 \pm 4.3 \%)$.

Few authors have provided data for peripapillary CC VD. Here, we obtained an overall mean of $60.8 \pm 5.3 \%$ across the different quadrants, and the higher VDs were observed in the nasal and temporal peripapillary quadrants (both $\geq 65 \%$ ). Similar values were reported by Wang et al. for 110 healthy

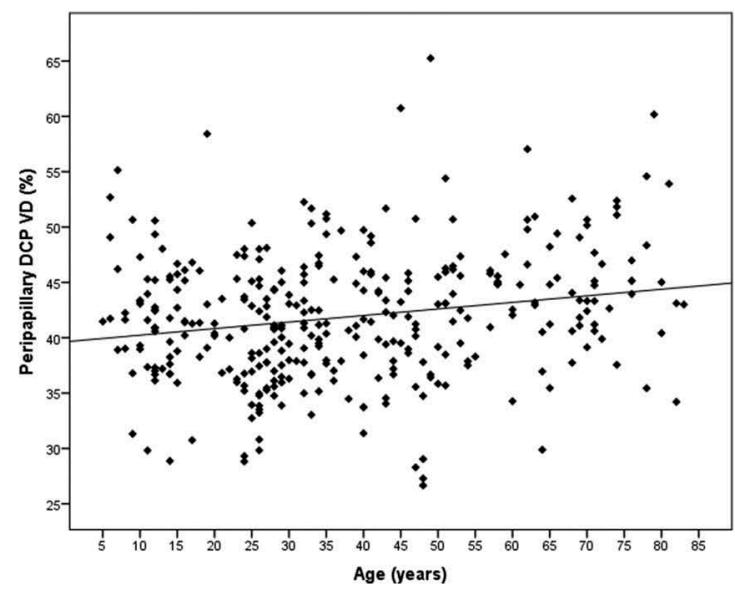

Figure 2. Correlations between peripapillary vessel density (VD) and age in the superficial capillary plexus (SCP) and deep capillary plexus (DCP) indicating that, with age, SCP VD decreases and DCP VD increases. 
participants (mean peripapillary CC VD $64.9 \pm 2.7 \%$ ). ${ }^{25}$ Contrasting with our findings, Yip et al. did not detect in 55 healthy participants using the RTVue XR Avanti a higher choroidal peripapillary VD in the horizontal than vertical quadrants (nasal and temporal: 0.236 \pm 0.094 and $0.192 \pm 0.101$ respectively, versus inferior and superior: $0.229 \pm 0.084$ and $0.260 \pm 0.101$ respectively) ${ }^{26}$ Future studies of CC peripapillary vasculature analyzing different sectors are needed to determine why CC showed higher VDs in the horizontal sectors. As an explanation, it could be that as the ciliary arteries run on each horizontal side of the optic nerve, when they divide, a greater number of short posterior ciliary arteries enter the horizontal sectors. Another possibility would be that small branches of the two long posterior ciliary arteries penetrate the sclera on both sides of the optic nerve, in the horizontal meridian, despite their main function being to nourish the choroid anteriorly.

We found no significant difference in peripapillary VD measurements between both eyes in a subgroup of individuals $(\mathrm{N}=33 ; \mathrm{P} \geq 0.139)$. Despite this, no correlation with the fellow eye was observed in any of the plexuses or quadrants examined $(\mathrm{R} \leq 0.137)$. Hou et al. observed $1.7 \%$ inter-eye peripapillary VD asymmetry in 55 healthy subjects and greater asymmetry at $2.2 \%$ in glaucoma patients $(P=.026){ }^{27}$

When we examined the impacts of different parameters on peripapillary $\mathrm{VD}$, correlation with age was negative in SCP and positive in DCP. In a recent review of determining factors for VD, Brücher et al. concluded that age is a strong determinant of retinal VD, and that the VD of the superficial vasculature diminishes with age. ${ }^{28}$ On the contrary, Bazvand et al. reported no significant differences in superficial peripapillary VD across different age groups $(\mathrm{N}=79$; range 12 to 67 years). ${ }^{11}$ However, they admitted that they anticipated some VD changes, albeit mild, during ageing but that their small sample size likely precluded detecting these changes. ${ }^{11}$ Interestingly in a study by Falavarjani et al. including 58 participants, similar positive correlation (as in our study in DCP) was observed between age and peripapillary $\mathrm{ONH}$ VD $(\mathrm{R}=0.277 ; P=.035)$ assessed in face images. $^{24}$ Zheng et al. showed that in normal aging, flow deficits in the CC increased with age. ${ }^{29}$ Interestingly, Orlov et al. described by fundus photography that three main traits (tortuosity, vessel bifurcation number, and vessel endpoint number) showed significant changes with age. ${ }^{30}$ Further studies are therefore needed to clarify the effects of age in the different plexuses.

We noted no gender differences in peripapillary VD in any of the plexuses or quadrants studied. Consistent with our results, Mansoori et al. found that gender had no effect on peripapillary $\operatorname{VD}(P=.7){ }^{19}$ In contrast, Bazvand et al. reported higher temporal and inferotemporal peripapillary VDs in women than men. ${ }^{11}$ Rao et al. also reported greater peripapillary VD in female subjects in the nasal and inferotemporal quadrants. ${ }^{8}$ However, as forewarned by these authors, these higher VDs in females require validation in future studies.

No correlation was found here between AL and retinal VD measured in the peripapillary region, as reported by Wen et al. for superficial VD measurements made using the same device as in our study in 75 healthy participants with a mean AL of $24.9 \pm 1.3 \mathrm{~mm}(21.8-28.8)(P=.904) .{ }^{31}$ However, we found negative correlation between CC VD and AL. This has been shown in some studies. ${ }^{28}$ We should highlight the study of Mastropasqua et al., who claimed that the total signal void area was significantly greater in myopic eyes than control eyes, and observed that the peripapillary region exhibited the greatest total signal void area $(p<.001$ vs macular region and peripheral retinal region). ${ }^{32}$

We found a significant correlation between disc area and CC VD in our multivariate analysis, but not for the SCP. Similarly, Falavarjani et al. were unable to correlate peripapillary retinal VD with disc area in normal eyes $(\mathrm{R}=0.204$; $P=.125) .{ }^{24}$ However, the different device employed should be considered, as they used an RTVue XR Avanti device with two concentric circles with 1.5 and $3.4 \mathrm{~mm}$ diameter centered on the disc, and the Triton uses an ETDRS grid with 1 and $3 \mathrm{~mm}$ respectively for the papillary and peripapillary subfields respectively.

According to the findings of Werner et al., OCTA may be particularly appropriate in very early or late-stage disease, when standard functional or structural diagnostic tests fail to offer useful data. ${ }^{4,5,10,14,15}$ Werner and Shen mentioned that a preceding structural and functional decline does not necessarily mean causation but that this may reflect the metabolic changes of "sick" retinal ganglion cells. ${ }^{5}$ In contrast, in more advanced glaucoma, RNFL measurements reach a plateau limiting the utility of detecting changes, and progression can be assessed only via VF assessment. Fortunately, peripapillary VD seems to have less of a floor effect than RNFL thickness. ${ }^{5,33}$

It should be highlighted that, as stated by many authors, absolute peripapillary VD values calculated with different algorithms are not directly interchangeable, as the dimensions of the area explored and the exact depth at which VD is assessed differ between instruments. ${ }^{5,9}$ This means that studies adopting VD as an outcome measure should not rely on external normative databases. ${ }^{9}$ Nonetheless, even with this lack of standardization, OCTA studies have provided important evidence of microvascular compromise in glaucoma. $6,7,14-18,22,23$ Hence, comparisons of measurements across OCTA devices and techniques should be considered with caution, and the same algorithm should be used for longitudinal monitoring of VD. Besides, differences between healthy and diseased eyes can be appreciated with different discriminatory abilities as a function of the plexus analyzed. ${ }^{7,9,33,34}$ In this regard, observations by Shin et al. indicate that although both SCP and DCP are sparser in glaucomatous eyes, only SCP VD shows comparable performance to RNFL thickness for a diagnosis of glaucoma, as these authors found that deep layer measurements were affected by $\mathrm{AL}$ and showed no relationship with glaucoma severity. ${ }^{7}$ Further, Liu et al. argue that the NFLP slab shows the best diagnostic accuracy for visualizing focal peripapillary perfusion defects, while the superficial vascular complex slab (SVC includes NFLP and GCLP) offers the best correlation with visual field. It is, therefore, possible that blood flow parameters are a better surrogate for function and better metric for monitoring glaucoma progression. ${ }^{33}$ 
Our study has several limitations. For peripapillary measurements, the examiner has to manually locate the measurement zone at the optic disk. Also, the segmented peripapillary analysis offered by the software does not separately identify NFLP and GCLP, and these were here measured together in the SCP. The software provided with OCTA devices should include a more detailed analysis of the peripapillary subfields, for example offering results for 6 or 12 sectors.

There is also great interindividual variability in optic disc configurations which could affect structural peripapillary measurements. However, inter-individual variability in the shape and size of the $\mathrm{ONH}$ affects peripapillary less than papillary VD quantification, so peripapillary measurements appear to offer more useful diagnostic information and have become the focus of most research in this area. ${ }^{5}$ The software employed here included the larger vessels in the density measurements. However, Liu et al. showed that capillary density (CD) excluding larger vessels offered slightly better diagnostic accuracy than VD including larger vessels (area under the receiver operating characteristic curve NFLP-CD $=0.947$ versus NFLP-VD $=0.934$; SVC-CD 0.942 versus SVC-VD 0.919). ${ }^{33}$ The authors claimed that both (CD and VD) showed excellent performance, and currently both parameters are considered to offer similar information. Ideally, it should be possible to remove the larger vessel in the analysis when studying the ONH VD.

In addition, a general limitation of VD quantification in OCTA devices is the projection artifact of the superficial layers over the deeper ones, which may affect the accuracy of measurements. Liu et al. developed a projection-resolved OCTA algorithm to effectively remove the projection artifact and provide clean angiograms of deeper vascular plexuses in the peripapillary area. ${ }^{33}$ This research group showed that SVC is the best slab to detect glaucomatous changes in the macular region. ${ }^{35}$ As previously described by Spaide et al., the problem of the slab based approach is that segmentation is needed and this segmentation may introduce errors. ${ }^{36}$ A further limitation was the inclusion of only Caucasian subjects, limiting the external applicability of results. Finally, several factors (e.g., motion artifacts, measurement and analysis methods, image quality, lack of fovea-disc axis correction, the device threshold for the detection of blood vessels or systemic factors such as blood pressure, diabetes or unknown diseases) may modify VD and affect the accuracy and repeatability of VD measurements. ${ }^{37-39}$ Recently, using the Triton device, we reported the moderate reproducibility of these measurements, as have others employing different OCTA devices. ${ }^{40,41}$ In our previous study, peripapillary VD measurements in the same setting showed an intraobserver ICC $\geq 0.499$ for all subfields and plexuses, with an interobserver ICC $\geq 0.620 .^{40}$ The highest ICC recorded were for intraobserver reproducibility in the SCP $(0.853)$ and CC (0.828). Interobserver agreement values were 0.870 for SCP and 0.868 for CC. The CV of the peripapillary measurements ranged from $4.8 \%$ to $16.4 \%$. Overall, VD reproducibility coefficients were higher for the ONH (ICC $\geq 0.499$ ) than macula (ICC $\geq 0.268$ ). Accordingly, these variation sources need to be minimized to ensure reliability between exams.

Our study offers the largest and most complete SS-OCTA normative database using the Triton device of peripapillary VD for the different retinal plexuses and the $\mathrm{CC}$, as useful reference ranges to interpret VD measurements. It also assesses the impacts of different factors in a large healthy Caucasian population, which will be useful for interpreting OCTA metrics and identifying and characterizing pathological changes in these structures. This large dataset of peripapillary VD measurements revealed wide individual variation and correlations with age. No associations emerged with gender, and only in the CC, was VD negatively correlated with AL and disc area.

\section{Disclosure statement}

The authors declare that they have no conflict of interest.

\section{Financial support}

No financial support was received for this submission.

\section{ORCID}

José Ignacio Fernández-Vigo (1) http://orcid.org/0000-0001-8745-3464

\section{References}

1. Hulsman CA, Vingerling JR, Hofman A, Witteman JC, de Jong PT. Blood pressure, arterial stiffness, and open-angle glaucoma: the Rotterdam study. Arch Ophthalmol. 2007;125 (6):805-12. doi:10.1001/archopht.125.6.805.

2. Memarzadeh F, Ying-Lai M, Chung J, Azen SP, Varma R. Blood pressure, perfusion pressure, and open-angle glaucoma: the Los Angeles Latino eye study. Invest Ophthalmol Vis Sci. 2010;51 (6):2872-77. doi:10.1167/iovs.08-2956.

3. Caprioli J, Coleman AL. Blood pressure, perfusion pressure, and glaucoma. Am J Ophthalmol. 2010;149(5):704-12. doi:10.1016/j. ajo.2010.01.018.

4. Jia Y, Wei E, Wang X, Zhang X, Morrison JC, Parikh M, Lombardi LH, Gattey DM, Armour RL, Edmunds B, et al. Optical coherence tomography angiography of optic disc perfusion in glaucoma. Ophthalmology. 2014;121(7):1322-32. doi:10.1016/j.ophtha.2014.01.021.

5. Werner AC, Shen LQ. A review of OCT angiography in glaucoma. Semin Ophthalmol. 2019;34(4):279-86. doi:10.1080/08820538.2019.1620807.

6. Scripsema NK, Garcia PM, Bavier RD, Chui TY, Krawitz BD, Mo S, Agemy SA, Xu L, Lin YB, Panarelli JF, et al. Optical coherence tomography angiography analysis of perfused peripapillary capillaries in primary open-angle glaucoma and normal-tension glaucoma. Invest Ophthalmic Vis Sci. 2016;57(9):OCT611-OCT620. doi:10.1167/ iovs.15-18945.

7. Shin JW, Sung KR, Lee JY, Kwon J, Seong M. Optical coherence tomography angiography vessel density mapping at various retinal layers in healthy and normal tension glaucoma eyes. Graefes Arch Clin Exp Ophthalmol. 2017;255(6):1193-202. doi:10.1007/s00417017-3671-4.

8. Rao HL, Pradhan ZS, Weinreb RN, Reddy HB, Riyazuddin M, Sachdeva S, Puttaiah NK, Jayadev C, Webers CAB. Determinants of peripapillary and macular vessel densities measured by optical coherence tomography angiography in normal eyes. J Glaucoma. 2017;26(5):491-97. doi:10.1097/IJG.0000000000000655.

9. Rabiolo A, Gelormini F, Sacconi R, Cicinelli MV, Triolo G, Bettin P, Nouri-Mahdavi K, Bandello F, Querques G. Comparison of methods to quantify macular and peripapillary vessel density in optical coherence tomography angiography. PLoS One. 2018;13(10):e0205773. doi:10.1371/journal.pone.0205773.

10. Yarmohammadi A, Zangwill LM, Diniz-Filho A, Suh MH, Yousefi S, Saunders LJ, Belghith A, Manalastas PI, Medeiros FA, Weinreb RN. Relationship between optical coherence tomography angiography vessel density and severity of visual field loss in 
glaucoma. Ophthalmology. 2016;123(12):2498-508. doi:10.1016/j. ophtha.2016.08.041.

11. Bazvand F, Mirshahi R, Fadakar K, Faghihi H, Sabour S, Ghassemi F. The quantitative measurements of vascular density and flow area of optic nerve head using optical coherence tomography angiography. J Glaucoma. 2017;26(8):735-41. doi:10.1097/ IJG.0000000000000722.

12. Mansoori T, Sivaswamy J, Gamalapati JS, Agraharam SG, Balakrishna N. Measurement of radial peripapillary capillary density in the normal human retina using optical coherence tomography angiography. J Glaucoma. 2017;26(3):241-46. doi:10.1097/ IJG.0000000000000594.

13. Wang Q, Chan S, Yang JY, You B, Wang YX, Jonas JB, Wei WB. Vascular density in retina and choriocapillaris as measured by optical coherence tomography angiography. Am J Ophthalmol. 2016;168:95-109. doi:10.1016/j.ajo.2016.05.005.

14. Triolo G, Rabiolo A, Shemonski ND, Fard A, Di Matteo F, Sacconi R, Bettin P, Magazzeni S, Querques G, Vazquez LE, et al. Optical coherence tomography angiography macular and peripapillary vessel perfusion density in healthy subjects, glaucoma suspects, and glaucoma patients. Invest Ophthalmol Vis Sci. 2017;58(13):5713-22. doi:10.1167/iovs.17-22865.

15. Akil H, Huang AS, Francis BA, Sadda SR, Chopra V. Retinal vessel density from optical coherence tomography angiography to differentiate early glaucoma, pre-perimetric glaucoma and normal eyes. PLoS One. 2017;12(2):e0170476. doi:10.1371/journal. pone.0170476.

16. Zhang S, Wu C, Liu C, Jia Y, Zhang Y, Zhang Y, Zhang H, Zhong Y, Huang D. Optical coherence tomography angiography of the peripapillary retina in primary angle closure glaucoma. Am J Ophthalmol. 2017;182:194-200. doi:10.1016/j.ajo.2017.07.024.

17. Rao HL, Kadambi SV, Weinreb RN, Puttaiah NK, Pradhan ZS, Rao DAS, Kumar RS, Webers CAB, Shetty R. Diagnostic ability of peripapillary \& vessel density measurements of optical coherence tomography angiography in primary open-angle and angleclosure glaucoma. Br J Ophthalmol. 2017;101(8):1066-70. doi:10.1136/bjophthalmol-2016-309377.

18. Suwan Y, Geyman LS, Fard MA, Tantraworasin A, Chui TY, Rosen RB, Ritch R. Peripapillary perfused capillary density in exfoliation syndrome and exfoliation glaucoma versus POAG and healthy controls: an OCTA study. Asia Pac J Ophthalmol. 2017;7(2):84-89.

19. Mansoori T, Sivaswamy J, Gamalapati JS, Balakrishna N. Topography and correlation of radial peripapillary capillary density network with retinal nerve fibre layer thickness. Int Ophthamol. 2018;38(3):967-74. doi:10.1007/s10792-017-0544-0.

20. Cennamo G, Montorio D, Velotti N, Sparnelli F, Reibaldi M, Cennamo G. Optical coherence tomography angiography in pre-perimetric open-angle glaucoma. Graefes Arch Clin Exp Ophthalmol. 2017;255(9):1787-93. doi:10.1007/s00417-017-3709-7.

21. Akagi T, Iida Y, Nakanishi H, Terada N, Morooka S, Yamada H, Hasegawa T, Yokota S, Yoshikawa M, Yoshimura N. Microvascular density in glaucomatous eyes with hemifield visual field defects: an optical coherence tomography angiography study. Am J Ophthalmol. 2016;168:237-49. doi:10.1016/j.ajo.2016.06.009.

22. Lommatzsch C, Rothaus K, Koch JM, Heinz C, Grisanti S. Vessel density in OCT angiography permits differentiation between normal and glaucomatous optic nerve heads. Int J Ophthalmol. 2018;11(5):835-43. doi:10.18240/ijo.2018.05.20.

23. Pinhas A, Linderman R, Mo S, Krawitz BD, Geyman LS, Carroll J, Rosen RB, Chui TY. A method for age-matched OCT angiography deviation mapping in the assessment of disease-related changes to the radial peripapillary capillaries. PLoS One. 2018;13 (5):e0197062. doi:10.1371/journal.pone.0197062.

24. Falavarjani KG, Shenazandi H, Naseri D, Anvari P, Sedaghat A, Hashemi M, Sadun AA. Correlation of optic disc morphometry and optic disc microvasculature assessed with optical coherence tomography angiography. Can J Ophthalmol. 2018;53(6):595-99. doi:10.1016/j.jcjo.2018.01.026.
25. Wang Q, Chan SY, Yang JY, You B, Wang YX, Jonas JB, Wei WB. Density of the macular and radial peripapillary capillary network measured by optical coherence tomography angiography. Acta Ophthalmol. 2017;95(6):e511-e512. doi:10.1111/aos.2017.95.issue-6.

26. Yip VCH, Wong HT, Yong VKY, Lim BA, Hee OK, Cheng J, Fu H, Lim C, Tay ELT, Loo-Valdez RG, et al. Optical coherence tomography angiography of optic disc and macula vessel density in glaucoma and healthy eyes. J Glaucoma. 2019;28(7):80-87. doi:10.1097/IJG.0000000000001125.

27. Hou H, Moghimi S, Zangwill LM, Shoji T, Ghahari E, Manalastas PIC, Penteado RC, Weinreb RN. Inter-eye asymmetry of optical coherence tomography angiography vessel density in bilateral glaucoma, glaucoma suspect, and healthy eyes. Am J Ophthalmol. 2018;190:69-77. doi:10.1016/j.ajo.2018.03.026.

28. Brücher VC, Storp JJ, Eter N, Alnawaiseh M. Optical coherence tomography angiography-derived flow density: a review of the influencing factors. Graefes Arch Clin Exp Ophthalmol. 2019 Dec 9. doi:10.1007/s00417-019-04553-2. [Epub ahead of print].

29. Zheng F, Zhang Q, Shi Y, Russell JF, Motulsky EH, Banta JT, Chu Z, Zhou H, Patel NA, de Sisternes L, et al. Age-dependent changes in the macular choriocapillaris of normal eyes imaged with swept-source optical coherence tomography angiography. Am J Ophthalmol. 2019;200:110-22. doi:10.1016/j.ajo.2018.12.025.

30. Orlov NV, Coletta C, van Asten F, Qian Y, Ding J, AlGhatrif M, Lakatta E, Chew E, Wong W, Swaroop A, et al. Age-related changes of the retinal microvasculature. PLoS One. 2019;14(5): e0215916. doi:10.1371/journal.pone.0215916.

31. Wen C, Pei C, Xu X, Lei J. Influence of axial length on parafoveal and peripapillary metrics from swept source optical coherence tomography angiography. Curr Eye Res. 2019;44(9):980-86. doi:10.1080/02713683.2019.1607393.

32. Mastropasqua R, Viggiano P, Borrelli E, Evangelista F, Libertini D, Di Antonio L, Toto L. In vivo mapping of the choriocapillaris in high myopia: a widefield swept source optical coherence tomography angiography. Sci Rep. 2019;9(1):18932. doi:10.1038/s41598-019-55192-w.

33. Liu L, Edmunds B, Takusagawa $\mathrm{H}$, Tehrani S, Lombardi L, Morrison JC, Jia Y, Huang D. Projection-resolved optical coherence tomography angiography of the peripapillary retina in glaucoma. Am J Ophthalmol. 2019;207:99-109. doi:10.1016/j. ajo.2019.05.024.

34. Suh MH, Zangwill LM, Manalastas PIC, Belghith A, Yarmohammadi A, Medeiros FA, Diniz-Filho A, Saunders LJ, Weinreb RN. Deep retinal layer microvasculature dropout detected by the optical coherence tomography angiography in glaucoma. Ophthalmology. 2016;123(12):2509-18. doi:10.1016/j. ophtha.2016.09.002.

35. Takusagawa HL, Liu L, Ma KN, Jia Y, Gao SS, Zhang M, Edmunds B, Parikh M, Tehrani S, Morrison JC, et al. Projectionresolved optical coherence tomography angiography of macular retinal circulation in glaucoma. Ophthalmology. 2017;124 (11):1589-99. doi:10.1016/j.ophtha.2017.06.002.

36. Spaide RF, Fujimoto JG, Waheed NK, Sadda SR, Staurenghi G. Optical coherence tomography angiography. Prog Retin Eye Res. 2018;64:1-55. doi:10.1016/j.preteyeres.2017.11.003.

37. Fenner BJ, Tan GSW, Tan ACS, Yeo IYS, Wong TY, Cheung GCM. Identification of imaging features that determine quality and repeatability of retinal capillary plexus density measurements in OCT angiography. Br J Ophthalmol. 2018;102 (4):509-14. doi:10.1136/bjophthalmol-2017-310700.

38. Pascual-Prieto J, Burgos-Blasco B, Avila-Sanchez-Torija M, Arriola-Villalobos P, Fernández-Vigo JI, Barbero-Pedraz MA, García-Feijóo J, Martínez-de-la-Casa JM. Utility of optical coherence tomography angiography in detecting vascular retinal damage caused by arterial hypertension. Eur J Ophthalmol. 2019:1120672119831159. doi:10.1177/1120672119831159.

39. Tuncer Z, Altuğ M. Does foveal position relative to the optic disc affect optical coherence tomography measurements in glaucoma? Turk J Ophthalmol. 2018;48(4):178-84. doi:10.4274/tjo. 
40. Fernández-Vigo JI, Kudsieh B, Macarro-Merino A, Arriola-Villalobos P, Martínez-de-la-Casa JM, García-Feijóo J, Fernández-Vigo JA. Reproducibility of macular and optic nerve head vessel density measurements by swept-source optical coherence tomography angiography. European J Ophthal. 2019:1120672119834472. doi:10.1177/1120672119834472.
41. She X, Guo J, Liu X, Zhu H, Li T, Zhou M, Wang F, Sun X. Reliability of vessel density measurements in the peripapillary retina and correlation with retinal nerve fiber layer thickness in healthy subjects using optical coherence tomography angiography. Ophthalmologica. 2018;240(4):183-90. doi:10.1159/000485957. 